\title{
December 2015 Pulmonary Case of the Month
}

\author{
Zachary M. Berg, MD \\ Kashif Yaqub, MD \\ Brian Wojek, MD \\ Khang Tran, MD \\ Karen L. Swanson, DO \\ Department of Pulmonary Medicine \\ Mayo Clinic Arizona \\ Scottsdale, AZ
}

\section{History of Present IIIness}

The patient is a 70-year-old man with a history of a chronic dry cough for 5 years, who presented to the emergency department with worsening cough and shortness of breath. Two weeks prior to symptom onset, was on trip in the United Kingdom, he developed gastroenteritis which spontaneously resolved.

\section{Past Medical History, Social History, and Family History}

- Old healed TB scar with positive PPD at 17 years of age prior to joining Air Force. No treatment given and patient was asymptomatic from a pulmonary point of view since then.

- Squamous cell carcinoma of the skin on the scalp, status post excision complicated by osteomyelitis, status post surgical graft from hip with prolonged course of IV antibiotics in 2010.

- Fractured left clavicle, status post repair 20 years ago.

- Hay fever.

- Hyperlipidemia.

- Squamous cell carcinoma removed from left arm.

- Varicose veins, lower extremity.

- Married. Retired police officer. Does not smoke.

- Family history is noncontributory

\section{Physical Examination}

- General: In moderate respiratory distress.

- Vitals: SpO2 on room air of $65 \%, 94 \%$ on high flow oxygen. Blood pressure 124/84, afebrile

- Lungs: Fine bibasilar crackles posteriorly.

- Heart: Regular rhythm without murmur.

- The remainder of the physical examination was normal.

\section{Laboratory Evaluation}


- CBC: unremarkable except white blood cell count $20.5 \times 10^{3}$ cells/yL, neutrophil predominant

- BNP: $366 \mathrm{pg} / \mathrm{mL}$

- Mycobacterium Quantiferon: Positive

- Mycoplasma IgM: Positive at $1.18 \mathrm{U} / \mathrm{L}$

\section{Radiography}

Initial chest x-ray is shown in Figure 1.

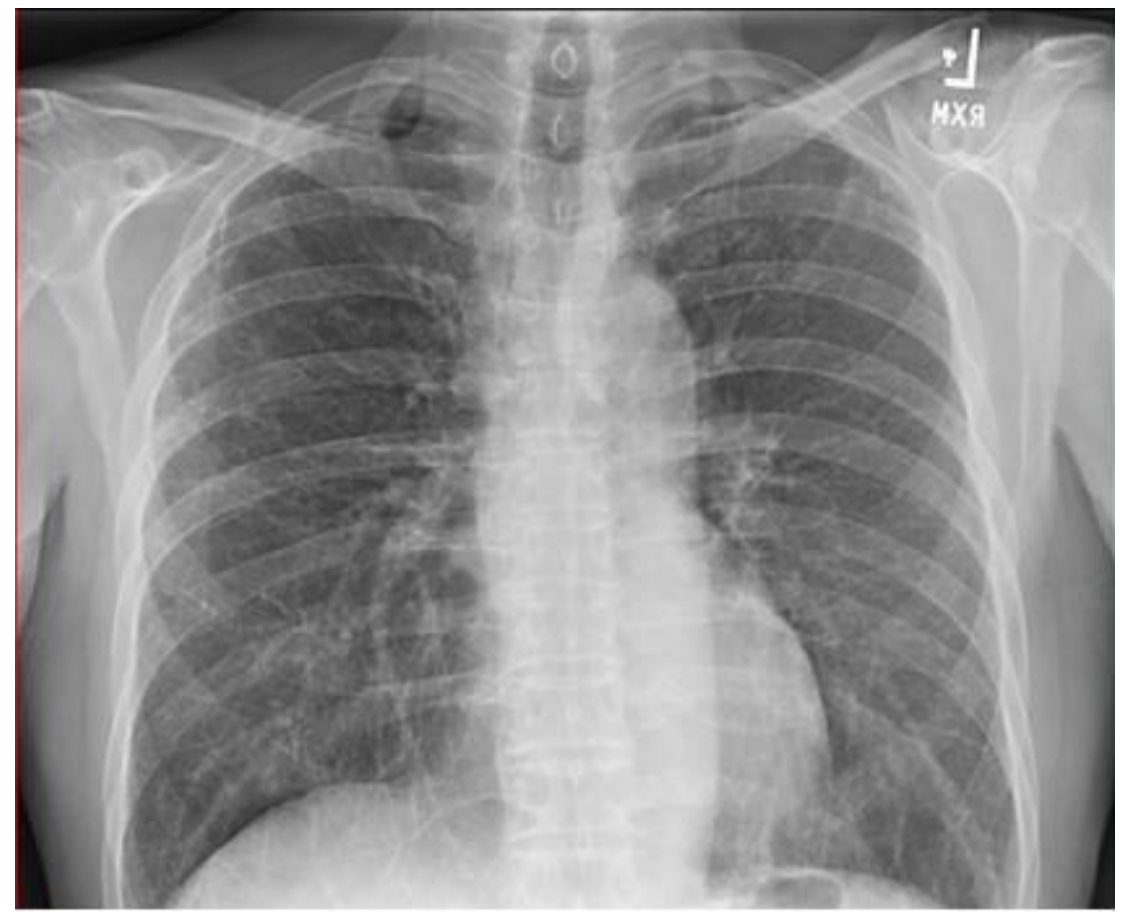

Figure 1. Initial chest x-ray.

What is the best next step in the patient's evaluation?

1. Begin erythromycin or doxycycline for Mycoplasma pneumonia

2. Begin heparin for presumptive pulmonary embolism

3. Thoracic CT scan

4. 1 and 3

5. All of the above 


\section{Correct! \\ 3. Thoracic CT scan}

There are at least two reasons to obtain a thoracic CT scan. First, the patient is profoundly hypoxic with an only mildly abnormal chest x-ray. This raises the possibility of an acute pulmonary embolus. The second is to better define the abnormalities seen on the chest x-ray. Unfortunately, Mycoplasma IgM is often falsely positive, and although it would not be wrong to empirically begin antibiotics, some skepticism should be maintained about the diagnosis (1). Similarly, it would not be wrong to begin to begin heparin for possible pulmonary embolism if there was a delay in obtaining a definitive study.

Representative views of the thoracic CT scan is shown in Figure 2.

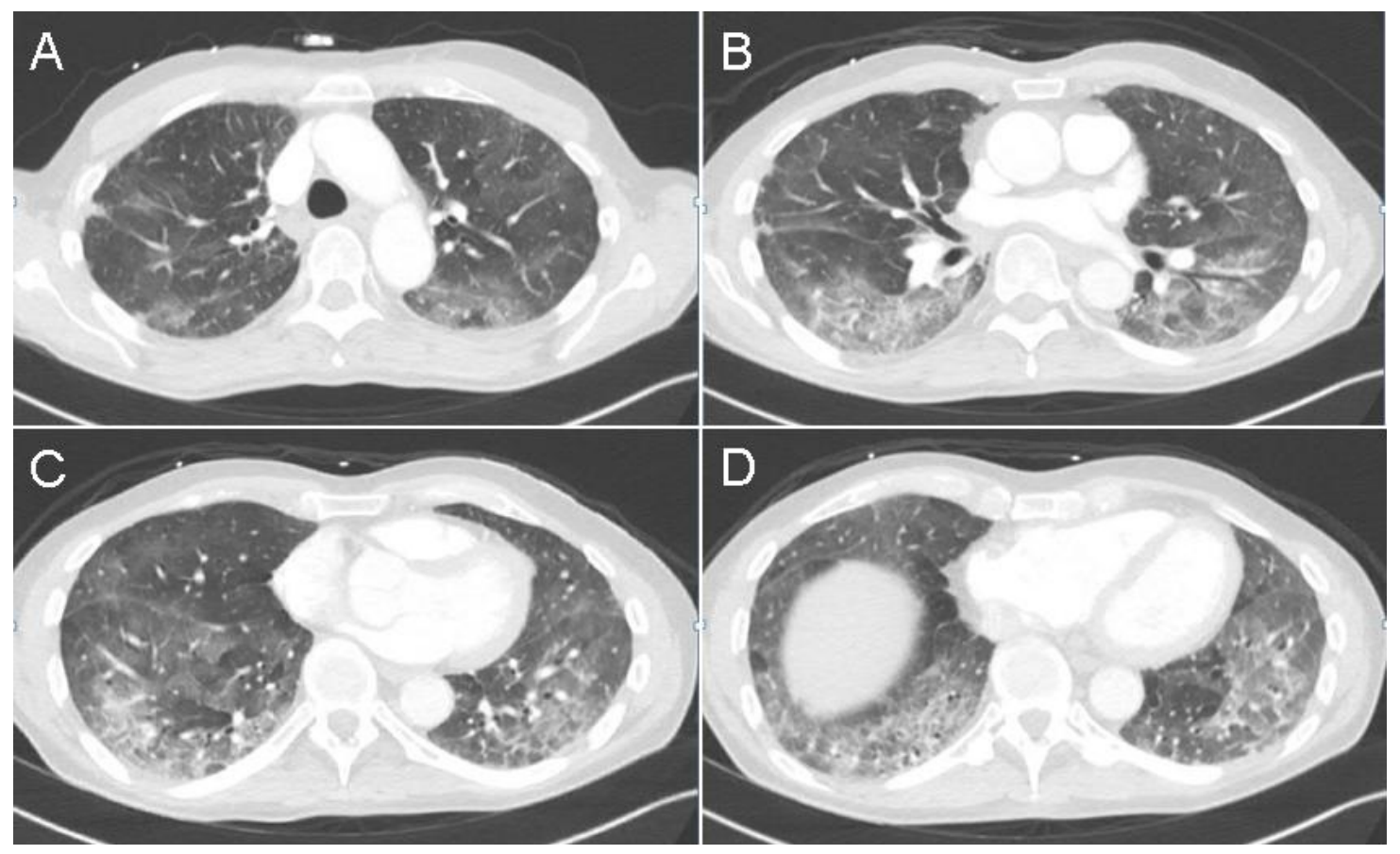

Figure 2. Representative images from the thoracic CT scan in lung windows.

The thoracic CT angiogram showed no pulmonary embolus. Sputum and blood cultures showed no growth.

What is the next step in the patient's evaluation?

1. Begin either nintedanib or pirfenidone

2. Begin high dosage corticosteroids

3. Bronchoscopy with bronchoalveolar lavage

4. Endobronchial ultrasound (EBUS) of mediastinal lymph nodes

5. Video-assisted thorascopic (VATS) lung biopsy 


\section{Correct!}

\section{Bronchoscopy with bronchoalveolar lavage}

There is still no established diagnosis. Since infection is still a leading possibility, bronchoscopy with bronchoalveolar lavage is reasonable. VATS is also reasonable depending on the expertise available and the clinical judgment of the clinician. Empiric treatment is not appropriate without an established or presumptive diagnosis. There are no enlarged lymph nodes on the CT scan.

Bronchoscopy with bronchoalveolar lavage was performed and the results are below:

- Normal bronchial anatomy

- Bronchoalveolar lavage differential

$>95 \%$ neutrophils

$>1 \%$ lymphocytes

$>4 \%$ alveolar macrophages

- Methicillin sensitive Staphylococcus aureus (MSSA)

- Candida albicans

- Aspergillus antigen 0.583 (normal < 0.5)

- No hemosiderin laden macrophages

- No malignant cells

Clinically it was thought that the MSSA, Candida and Aspergillus were all unlikely to explain the patient's overall picture although the patient was begun on methicillin and voriconazole. There was no clinical response.

What is the most likely diagnosis?

1. Acute hypersensitivity pneumonia

2. Acute interstitial pneumonia (Hamman Rich syndrome)

3. Adult respiratory distress syndrome

4. Lupus pneumonitis

5. Mycoplasma pneumonia 


\section{Correct! \\ 2. Acute interstitial pneumonia (Hamman Rich syndrome)}

In 1935 Hamman and Rich (2) described a disorder of rapidly progressive pneumonia that was not infectious or tuberculosis. This rare syndrome is characterized by rapidly progressive pulmonary fibrosis, leading to frequent confusion with idiopathic pulmonary fibrosis (3). In fact, the eponym Hamman Rich syndrome became synonymous with idiopathic pulmonary fibrosis despite clear differences in clinical presentation, radiography, pathology, and survival. In 1986, Katzenstein described eight patients with acute respiratory failure of unknown etiology. On biopsy, organizing diffuse alveolar damage was present in all specimens. Katzenstein coined the phrase acute interstitial pneumonitis (AIP) to distinguish it from the fibroproliferative stage of the acute respiratory distress syndrome (ARDS), which has an identical pathology. Since then, little progress into understanding this disease has been made. Treatment with corticosteroids is usually recommended although data supporting their use is weak.

Our patient was begun on corticosteroids but had little clinical improvement. About three weeks in to hospitalization his oxygenation not improving when he had a sudden clinical deterioration with dyspnea and hypoxia. The thoracic CT scan was repeated (Figure 3).

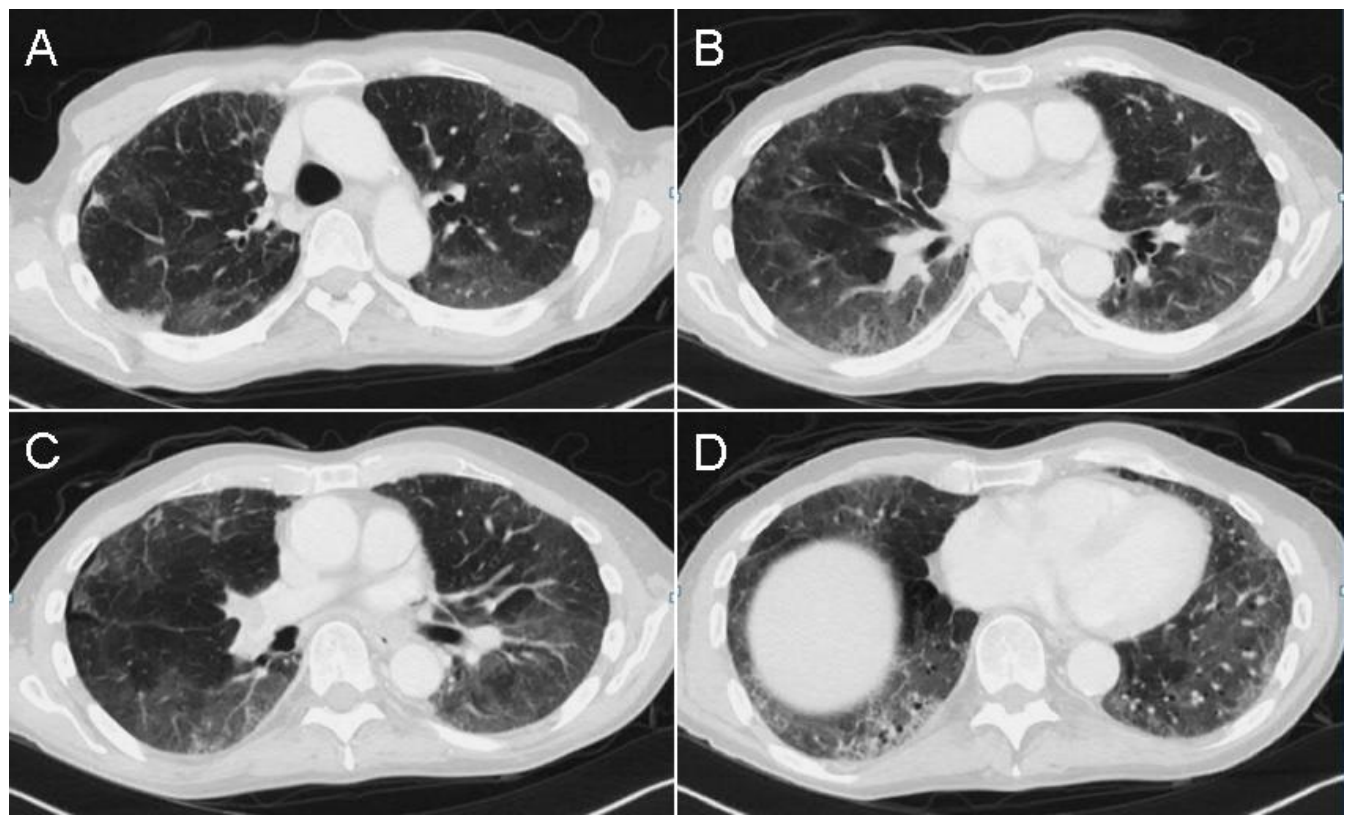

Figure 3. Representative views from repeat thoracic CT scan.

What new finding is present on the repeat thoracic CT scan?

1. Development of worsening ground glass opacities

2. Diffuse nodular disease

3. Increasing pulmonary fibrosis

4. Pneumothorax

5. Pulmonary embolus 


\section{Correct! \\ 5. Pulmonary embolus}

Although subtle, a filling defect in the right pulmonary artery is present in Figure $3 \mathrm{C}$ (Figure 4, arrow).

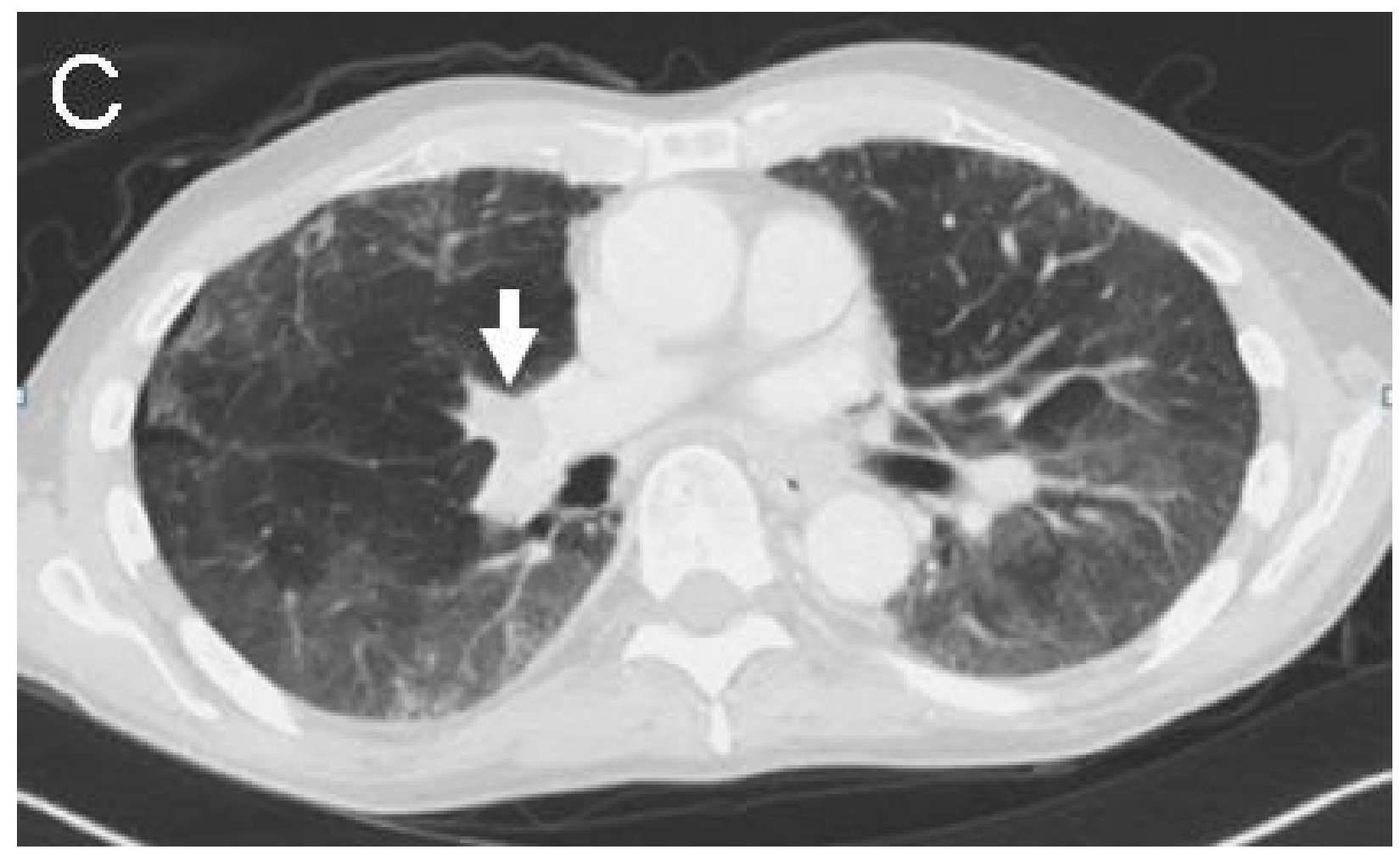

Figure 4. Enlarged view of Figure $3 \mathrm{C}$ showing right pulmonary artery filling defect. Note the lack of contrast in the vessels distal to the filling defect.

The patient was treated with anticoagulants and is currently being evaluated for a lung transplant.

\section{References}

1. Smith TF. Mycoplasma pneumoniae infections: diagnosis based on immunofluorescence titer of IgG and IgM antibodies. Mayo Clin Proc. 1986;61:8301. [CrossRef] [PubMed]

2. Hamman $L$, Rich AR. Fulminating diffuse interstitial fibrosis of the lungs. Trans Am Clin Climatol Assoc. 1935;51:154-63. [PubMed]

3. Vourlekis JS, Brown KK, Schwarz MI. Acute interstitial pneumonitis: current understanding regarding diagnosis, pathogenesis, and natural history. Semin Respir Crit Care Med. 2001;22(4):399-408. [CrossRef] [PubMed] 